\title{
A common model for the breathlessness experience across cardiorespiratory
} disease

\author{
Sarah L. Finnegan (1) ${ }^{1}$, Kyle T.S. Pattinson ${ }^{1}$, Josefin Sundh $\mathbb{1}^{2}{ }^{2}$, Magnus Sköld ${ }^{3,4}$, \\ Christer Janson $\mathbb{1}^{5}$, Anders Blomberg ${ }^{6}$, Jacob Sandberg $\mathbb{1}^{7}$ and \\ Magnus Ekström (D) $^{7}$
}

Affiliations: 'Wellcome Centre for Integrative Neuroimaging and Nuffield Division of Anaesthetics, Nuffield Dept of Clinical Neurosciences, University of Oxford, Oxford, UK. ${ }^{2}$ Dept of Respiratory Medicine, Faculty of Medicine and Health, School of Medical Sciences, Örebro University, Örebro, Sweden. ${ }^{3}$ Respiratory Medicine Unit, Dept of Medicine Solna and Center for Molecular Medicine, Karolinska Institutet, Stockholm, Sweden. ${ }^{4}$ Dept of Respiratory Medicine and Allergy, Karolinska University Hospital, Stockholm, Sweden. ${ }^{5}$ Dept of Medical Sciences: Respiratory, Allergy and Sleep Research, Uppsala University, Uppsala, Sweden. ${ }^{6}$ Dept of Public Health and Clinical Medicine, Section of Medicine, Umeå University, Umeå, Sweden. ${ }^{7}$ Respiratory Medicine and Allergology, Dept of Clinical Sciences, Faculty of Medicine, Lund University, Lund, Sweden.

Correspondence: Sarah L. Finnegan, Nuffield Dept of Anaesthetics and Oxford Centre for Functional Magnetic Resonance Imaging of the Brain, University of Oxford, 6th Floor, West Wing, John Radcliffe Hospital, Oxford, OX3 9DU, UK. E-mail: sarah.finnegananden.ox.ac.uk

ABSTRACT Chronic breathlessness occurs across many different conditions, often independently of disease severity. Yet, despite being strongly linked to adverse outcomes, the consideration of chronic breathlessness as a stand-alone therapeutic target remains limited. Here we use data-driven techniques to identify and confirm the stability of underlying features (factors) driving breathlessness across different cardiorespiratory diseases.

Questionnaire data on 182 participants with main diagnoses of asthma (21.4\%), COPD (24.7\%), heart failure (19.2\%), idiopathic pulmonary fibrosis (18.7\%), other interstitial lung disease (2.7\%), and "other diagnoses" (13.2\%) were entered into an exploratory factor analysis (EFA). Participants were stratified based on their EFA factor scores. We then examined model stability using 6-month follow-up data and established the most compact set of measures describing the breathlessness experience.

In this dataset, we have identified four stable factors that underlie the experience of breathlessness. These factors were assigned the following descriptive labels: 1) body burden, 2) affect/mood, 3) breathing burden and 4) anger/frustration. Stratifying patients by their scores across the four factors revealed two groups corresponding to high and low burden. These two groups were not related to the primary disease diagnosis and remained stable after 6 months.

In this work, we identified and confirmed the stability of underlying features of breathlessness. Previous work in this domain has been largely limited to single-diagnosis patient groups without subsequent retesting of model stability. This work provides further evidence supporting disease independent approaches to assess breathlessness.

@ERSpublications

Using machine learning techniques, four underlying factors of breathlessness in cardiorespiratory disease were identified. These underlying factors of the breathlessness experience were similar across diseases and remained stable over time. https://bit.ly/3brtD8c

Cite this article as: Finnegan SL, Pattinson KTS, Sundh J, et al. A common model for the breathlessness experience across cardiorespiratory disease. ERJ Open Res 2021; 7: 00818-2020 [https:// doi.org/10.1183/23120541.00818-2020].

This article has supplementary material available from openres.ersjournals.com

Received: 3 Nov 2020 | Accepted: 15 Feb 2021

Copyright (CThe authors 2021. This version is distributed under the terms of the Creative Commons Attribution NonCommercial Licence 4.0. For commercial reproduction rights and permissions contact permissions@ersnet.org 


\section{Introduction}

Chronic breathlessness, breathlessness persisting despite optimal treatment, is a central symptom in many conditions, especially in respiratory and cardiac diseases, but also in cancer, neurological diseases and for survivors of coronavirus disease 2019 (COVID-19) [1,2]. Breathlessness is strongly linked to poorer clinical outcomes, including worse quality of life and increased rates of anxiety and depression [1, 3-7]. While cardiorespiratory physiological mechanisms undoubtably often play a key role in breathlessness, they fail to explain breathlessness in many situations, such as when two individuals with objectively similar disease severities report very different experiences of breathlessness $[4,8]$. These discrepancies, alongside the multifaceted and subjective nature of chronic breathlessness, make its assessment and treatment challenging.

A multitude of assessment tools exist to quantify breathlessness. However, the focus of much recent research has been on identifying the "best" measurement tool rather than underlying features (or factors) and their relationship in driving the experience of breathlessness. Where domains of breathlessness have been explored, efforts have typically centred around distinctions between physical sensations of breathlessness i.e. work effort/air hunger, and the comparison of subjective intensity with unpleasantness [9-12]. While a deeper understanding of the sensation of breathlessness itself is clearly important, symptoms need to be contextualised within a person's broader lived experience. For example, clinical guidelines include "affective distress" and "symptom impact/burden" as domains of dyspnoea management [3], highlighting the relevance of anxiety, depression and fatigue, which may be addressed with mind-body interventions [13].

Multi-dimensional, data-driven models offer an opportunity to explore the bi-directional impact of breathlessness on both body and mind, revealing underlying "hidden" factors of breathlessness not previously considered. These underlying factors may not only form the basis of a common descriptive framework for breathlessness across cardiorespiratory disease but could enrich understanding of discordant breathlessness and become key therapeutic targets in their own right. Machine-learning techniques have already been used to identify baseline factors, which together predict treatment response in depression [14, 15] and pain [16, 17]. Similar approaches have been used to identify symptom-based phenotypes in asthma [18] and COPD [19, 20] where clusters of patients for whom breathlessness was linked with underlying mood and affect were identified. The findings of those studies supports our own previous findings, which revealed separable factors centring around mood and affect measures [21-23] and symptom burden measures [21, 22], with further important factors including anticipated and physical capability measures [22]. However, this work focused on identifying factors underling breathlessness within a single disease [22] or between a patient and a control group $[21,23]$. The aim of the present study was to address some outstanding questions:

1. Does a shared description of breathlessness exist across disease diagnoses?

2. Do weightings on any identified factors predict primary disease diagnosis?

3. Are factors stable across time?

\section{Methods}

This was an analysis of data from a longitudinal study of patients suffering from cardiorespiratory disease and breathlessness in everyday life (approved by the Regional Ethical Board at Lund University (DNr: 2016/16). This body of work uses the dataset of which parts were used in published validation of the Swedish Multidimensional Dyspnea Profile (MDP) [24], the Dyspnoea-12 [25], and the instruments' clinical feasibility and minimal clinically important differences [26]. The present analyses are novel and not previously reported.

\section{Participants}

182 participants (97 female, median age 72 years (range 19-91 years), asthma (21.4\%), COPD (24.7\%), heart failure (19.2\%), idiopathic pulmonary fibrosis (18.7\%), other interstitial lung disease $(2.7 \%)$, and "other diagnoses" including depression, cancer, diabetes and renal failure (13.2\%); table 1) were recruited from five outpatient clinics [24]. Inclusion criteria were: age 18 years or older, documented physician-diagnosed chronic cardiorespiratory disease, self-reported breathlessness during daily life defined as an answer "yes" to the question "Did you experience any breathlessness during the last 2 weeks?" and ability to give written informed consent to participate in the study. Exclusion criteria were: inability to write or understand Swedish adequately to participate, cognitive or other inability to participate in the study, or estimated survival of less than 3 months. Of the 182 participants who completed the baseline visit, 144 (79\%) provided follow-up data at six months (79 female, median age 72 years (range $20-92$ years)).

\section{Measures}

Participants attended the clinic for a baseline visit, while repeat data were collected six months after the first visit date via a postal questionnaire. Baseline data included demographics, smoking status, measured 
TABLE 1 Demographic information per main cause of breathlessness (total subjects=182)

\begin{tabular}{|c|c|c|c|c|}
\hline Main cause of breathlessness & Attended at baseline & Age years & Female & $\mathrm{BMI} \mathrm{kg} \cdot \mathrm{m}^{-2}$ \\
\hline COPD & $45(24.7 \%)$ & $71.6 \pm 9.2$ & 29 & $26.6 \pm 7.8$ \\
\hline Asthma & $39(21.4 \%)$ & $53.2 \pm 15.2$ & 29 & $27.9 \pm 5.3$ \\
\hline Heart failure & $35(19.2 \%)$ & $76.4 \pm 7.9$ & 8 & $30.5 \pm 5.7$ \\
\hline IPF & $34(18.7 \%)$ & $75.1 \pm 6.8$ & 16 & $25.9 \pm 3.9$ \\
\hline Other ILD & $5(2.7 \%)$ & $69.3 \pm 11.7$ & 2 & $28.4 \pm 4.5$ \\
\hline Other diagnoses ${ }^{\#}$ & $24(13.2 \%)$ & $66.4 \pm 14.6$ & 13 & $25.9 \pm 6.7$ \\
\hline $\begin{array}{l}\text { Number of patients with } 1 / 2 / 3 / 4 \\
\text { comorbidities }\end{array}$ & & $50 / 16 / 4 / 1$ & & \\
\hline $\begin{array}{l}\text { Comorbidity frequency by } \\
\text { diagnosis }\end{array}$ & & & & \\
\hline COPD & \multicolumn{4}{|c|}{$\begin{array}{l}\text { Diabetes } n=30 \text {; heart failure } n=10 \text {; other breathlessness } n=7 \text {; } \\
\text { asthma } n=5 \text {; cancer } n=4 \text {; renal failure } n=2\end{array}$} \\
\hline Asthma & \multicolumn{4}{|c|}{ Diabetes $n=8$; other breathlessness $n=2 ;$ ILD $n=2$} \\
\hline Heart failure & \multicolumn{4}{|c|}{$\begin{array}{l}\text { Diabetes } n=13 \text {; renal failure } n=8 ; \operatorname{COPD} n=4 ; \\
\text { other breathlessness } n=2 \text {; asthma } n=2 \text {; } \text { cancer } n=2\end{array}$} \\
\hline IPF & \multicolumn{4}{|c|}{$\begin{array}{l}\text { Diabetes } n=5 \text {; renal failure } n=2 \text {; other breathlessness } n=2 \text {; } \\
\text { heart failure } n=1 \text {; asthma } n=1\end{array}$} \\
\hline Other ILD & \multirow{2}{*}{\multicolumn{4}{|c|}{$\begin{array}{l}\text { Diabetes } n=2 ; \operatorname{COPD} n=1 \text {; heart failure } n=1 \\
\text { Heart failure } n=5 ; \text { diabetes } n=5 ; \text { asthma } n=3 \text {; renal failure } n=2 \text {; } \\
\text { cancer } n=1 ; \operatorname{COPD} n=1\end{array}$}} \\
\hline Other diagnoses $\#$ & & & & \\
\hline \multicolumn{5}{|c|}{$\begin{array}{l}\text { Data are presented as mean } \pm S D, n(\%) \text { or } n \text {. IPF: idiopathic pulmonary fibrosis; ILD: interstitial lung } \\
\text { disease; BMI: body mass index. }{ }^{\#} \text { : other diagnoses include depression, cancer, diabetes and renal failure. }\end{array}$} \\
\hline
\end{tabular}

height and weight, and self-report questionnaires, which were scored according to their respective manuals and recorded as their appropriate domain scores: COPD Assessment Test (CAT) [27]; Dyspnea-12 (D12) [28]; EuroQol Five Dimensions, Five Levels (EQ-5D-5L) [29]; Functional Assessment of Chronic Illness Therapy (FACIT)-Fatigue Scale; Hospital Anxiety and Depression Scale (HADS) [30]; Multidimensional Dyspnea Profile (MDP) [9]; modified Medical Research Council (mMRC) Breathlessness Scale [31]. Average severity of pain (0-10 numerical rating scale (NRS)) and average severity of breathlessness were measured as "on average during the last two weeks" (Likert scale), along with current severity of breathlessness (0-10 NRS).

Six months after their first visit participants were asked to complete and return a postal questionnaire pack. Questionnaires remained the same as at baseline and participants were asked to additionally rate their change in breathlessness since the first assessment on a seven-point ordinal scale (Global Impression of Change (GIC); where 1="very much better", 4="no change" and 7="very much worse") [26].

Analysis

A brief summary of analyses are provided here. Further information on technical details can be found within Supplementary materials.

\section{Factor identification}

Exploratory factor analysis (EFA) was used to identify and formalise any common structure underlying responses across clusters of questionnaire measures (table 2). EFA is a model-free process, allowing researchers to examine a dataset without applying a preconceived structure to the result [32-34]. This is sometimes called "unsupervised machine learning". In EFA, measures are grouped or discarded depending on how much they contributed to any one cluster. The resulting composite scores of each group are classed as a factor. Models were fit using Lavaan version 0.6-1 in R Studio version 1.2.1.

\section{Participant stratification}

Following exploratory factor analysis, each participant received a score (similar to the first component of a principal component analysis across measures within a factor) corresponding to each latent factor. To examine whether natural groupings of participants existed, the participants were stratified based on their factor scores using hierarchical cluster modelling techniques $[35,36]$. Hierarchical models were used to reorder participants based on their correlation strengths [35]. Models were programmed using Matlab (MATLAB 2018b, Mathworks, Natick, MA, USA). To determine whether the hierarchical groupings corresponded to disease diagnosis, the percentage probability of each of the disease categories was 


\section{TABLE 2 A list of measures included in the exploratory factor analysis model}

1. Sex

2. Age

3. $\mathrm{BMI}$

4. Dyspnea-12 - Affect

5. Dyspnea-12 - Physical

6. MDP A2 Anxiety

7. MDP_A2_Afraid

8. MDP_A2_Angry

9. MDP_A2_Frustrated

10. Breathlessness at rest

11. Breathlessness mean

12. HADS - Depression

13. HADS - Anxiety
14. $\mathrm{mMRC}$

15. EQ-5D-5L - Index

16. FACIT - Fatigue Scale

17. Average severity of pain

18. CAT

19. MDP_A2_Depression

20. MDP_A1_Breathing Discomfort

21. MDP_SQ1_Physical Effort

22. MDP_SQ2_Hunger

23. MDP_SQ3_Tightness

24. MDP SQ4_Mental Effort

25. MDP_SQ5_Hypernoea

BMI: body mass index; MDP: multidimensional dyspnea profile; SQ: sensory dimension; A2: emotional response domain; HADS: hospital anxiety and depression scale; FACIT: functional assessment of chronic illness therapy - fatigue scale; mMRC: modified Medical Research Council breathlessness scale; CAT: COPD assessment test; EQ-5D-5L: EuroQol five dimensions - five levels.

calculated for each group proportional to group size and compared with chance as shown in Supplementary equation (1).

\section{Longitudinal model validation}

To determine whether the exploratory factor analysis model established at baseline was stable six months later we re-examined the factor structure using a confirmatory factor analysis on the follow-up data. Model fit criteria compared the proposed model to a null model.

\section{Model refinement}

Following the generation of the factor model we assessed whether low loading items could be removed from the model while maintaining a significant model fit. The process was carried out iteratively and after each item's removal the model fitting procedure was rerun and assessed for significance using the above model selection criteria. Items were removed until the model no longer significantly fit.

\section{Results}

Establishing a shared description of breathlessness across disease diagnoses

Of the 25 measures entered into the exploratory factor analysis (table 2), 16 fulfilled the model fit criteria and were retained for model validation. The measures retained were: the EQ-5D-5L index, average severity of pain (0-10 NRS), (FACIT)-Fatigue Scale, CAT total score, HADS anxiety, mMRC, D12 physical, current and average severity of breathlessness (0-10 NRS), MDP A1 (breathing discomfort), MDP A2 (frustration, anger, fear, anxiety and depression), MDP SQ4 intensity (mental breathing effort). The composite scores of the 16 retained measures formed four factors, which were validated according to the model fit criteria (TLI=0.97, root mean square error of approximation (RMSEA) $=0.055$, SRMR=0.03), shown in figure 1. We assigned descriptive labels to each of the factors as follows:

- Factor 1: body burden: comprised EQ-5D-5L-index, pain and fatigue measures, CAT (a disease impact measure), and the SQ4 domain of the MDP (mental breathing effort).

- Factor 2: affect/mood: was composed of the anxiety, fear and depression A2 domains of the MDP along with the anxiety subscale of HADS.

- Factor 3: breathing burden: was made up of breathlessness at rest and on average, the mMRC, A1 domain of the MDP and the physical domain of the D12.

- Factor 4: anger/frustration: consisted of two of the A2 domains of the MDP - anger and frustration.

Factors 1 and 3, and factors 2 and 4 showed the strongest covariance (curved lines figure 1) but retained sufficient independence to not collapse into the same factor.

\section{Do weightings on any identified shared factors predict disease diagnosis?}

Participants were stratified based on their four composite factor scores from the EFA model fit. A two-group solution was confirmed by MATLAB's evalcluster algorithm as the most distinct and largely seems to correspond to high and low load across the four factors (figure 2). 
a)

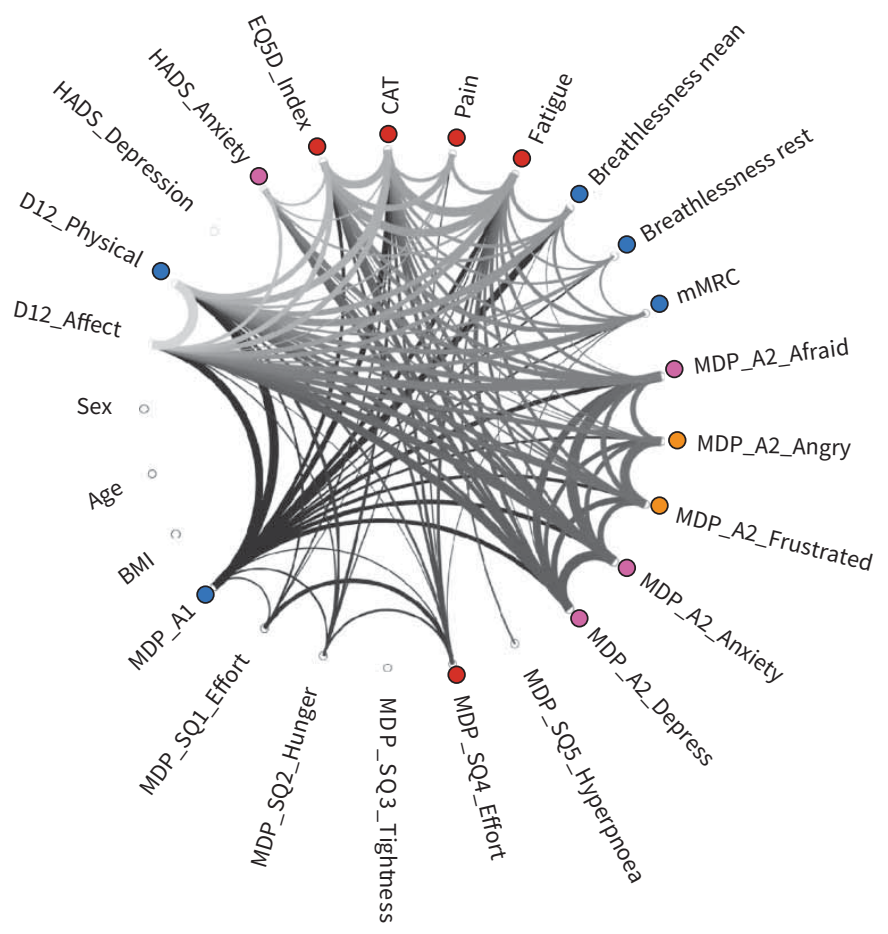

b)

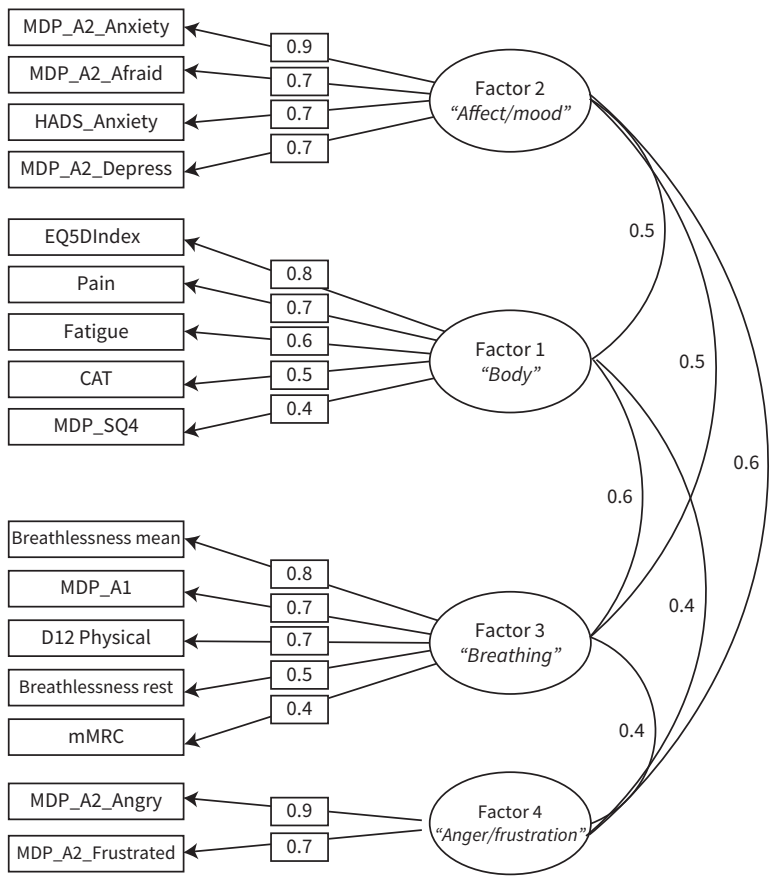

FIGURE 1 a) Connectogram. Measures are plotted as a wheel with connections between each measure input to the model shown as a thread. The thickness of the thread provides a visual representation of the strength of the relationship between any two measures ( $R \geqslant 0.35)$. Coloured dots highlight the common factors underlying groups of measures. Pink dots correspond to Factor 2 (affect/mood), red to Factor 1 (body burden), blue to Factor 3 (breathing burden) and orange to Factor 4 (anger/frustration). b) Factor diagram. The retained measures are shown on the left hand side of the diagram while the contribution of each measure to the resulting factor is shown as a loading weight (rectangular boxes) Factors are shown as ovals with descriptive labels underneath. The covariance across factors is illustrated by the curved lines.

The two groups were not found to correspond to primary disease diagnosis (figure 3), of which there were six categories: asthma, COPD, heart failure, idiopathic pulmonary fibrosis (IPF), other interstitial lung disease and "other" (including depression, cancer, diabetes and renal failure). However, participants with IPF, other interstitial lung disease or heart failure were more likely when compared to chance $(65 \%, 60 \%$ and $61 \%$ respectively) to be classified into the lower symptom burden group. While participants with COPD, asthma or a diagnosis of "other" were more likely to be classified into the higher symptom burden group when compared to chance (43\%, 50\% and $47 \%$ respectively).

\section{Assessing the stability of factors over time}

After six months, the factor model was found to have remained stable according to the confirmatory factor analysis model fit criteria (TLI=0.92, RMSEA=0.086 (marginal fit), SRMR=0.06).

\section{Establishing the simplest informative model}

The baseline model was then subjected to an iterative process in which the lowest loading variables were removed. After each cycle of variable removal, the model was retested. Figure 4 illustrates the final "compact" model. The MDP SQ4 (mental breathing effort) was removed along with mMRC, breathlessness at rest and CAT. The final model was found to be significant according to the model fit criteria (TLI=0.96, RMSEA=0.06, SRMR=0.03), and was found to remain stable after six months (TLI=0.93, RMSEA=0.084, SRMR=0.05), although RMSEA was considered to be marginal.

\section{Discussion}

We aimed to answer the following questions: 1) does a shared description of breathlessness exist across disease diagnoses? 2) do weightings on any identified factors predict primary disease diagnosis? 3) are factors stable across time? Our findings go beyond our previous work by showing that underlying factors of the breathlessness experience are similar across diseases and remain stable over time. Using unsupervised machine learning techniques, we identified four key factors underlying the experience of patients with chronic breathlessness. We assigned the key factors the following descriptive labels: body 


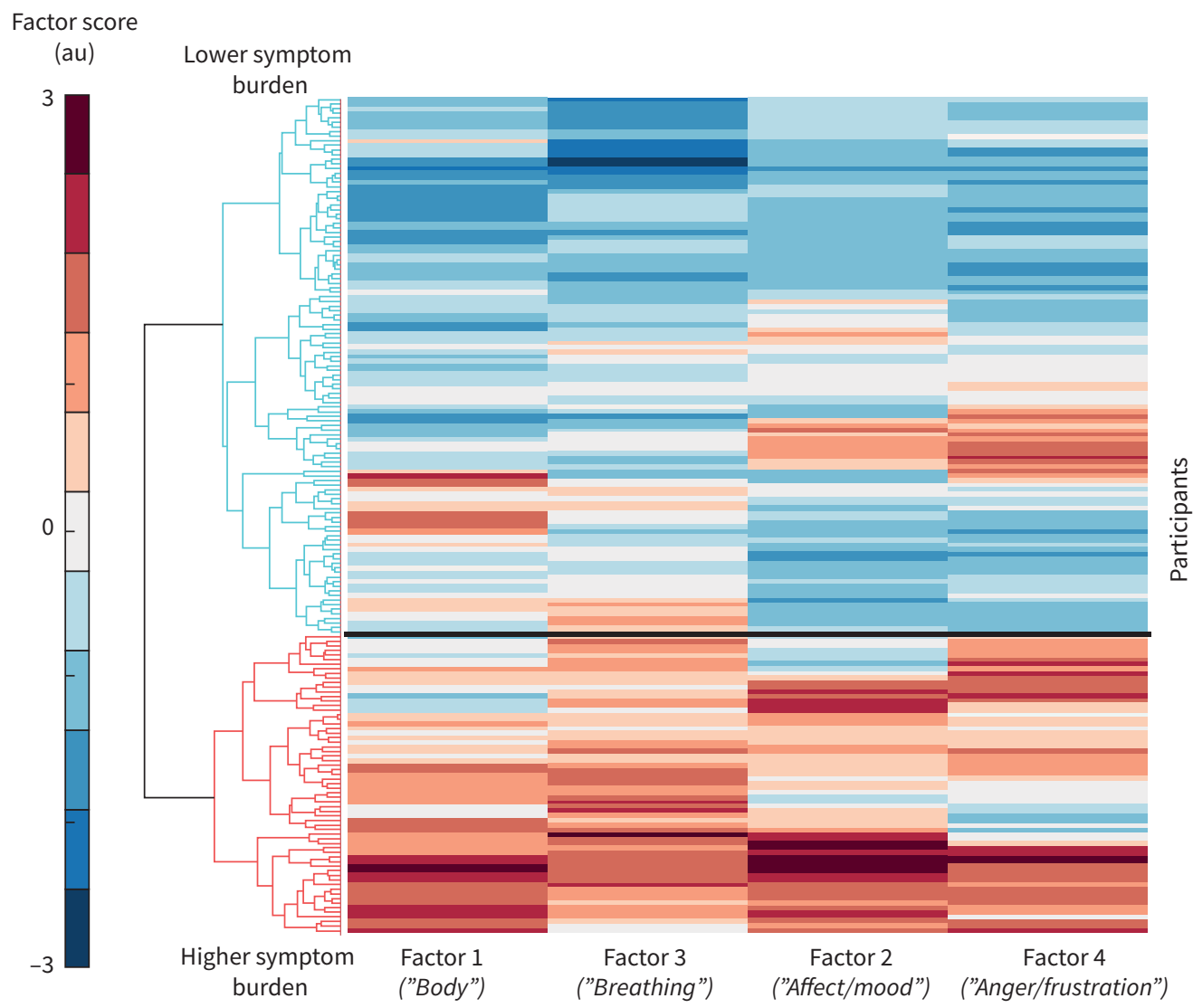

FIGURE 2 Clustergram. A matrix of each participants score across the four factors. Factor score is measured in arbitrary units (au). Each of the four factors is displayed along the bottom, with participants forming the $y$-axis. Factors 1 and 3 correspond to body and breathing burden, while Factors 2 and 4 correspond to affect/ mood and anger/frustration. A dendrogram is displayed along the left side and highlights the division of participants into the two groups, higher symptom burden and lower symptom burden.

burden, affect/mood, breathing burden and anger/frustration. Together these factors provide a common description of breathlessness across asthma, COPD, heart failure, idiopathic pulmonary fibrosis and other interstitial lung disease. These factors were found to be stable across time but were not predictive of the primary disease category. Instead participants fell into either high or low scorers across the four factors.

\section{Applying machine learning techniques to investigate breathlessness}

In this study, we used unsupervised machine learning techniques, a benefit of which is that hypotheses and relationships can be led by the data to reveal associations not previously considered [32, 36]. This exploratory approach does not, however, guarantee a statistically or clinically significant finding. Measures may have too little, or alternatively too much in common with all other measures to form separable factors. An example is the D12 affective score, which was removed from this model as it contributed strongly to both Factor 2 (mood/affect) and Factor 3 (breathing burden). In contrast the COPD assessment test was retained, suggesting that the constructs assessed are common across different cardiorespiratory conditions. These considerations lend confidence to our findings but reinforce the caveats of these techniques: factor analysis builds models based on shared variance and requires linear relationships between variables. Excluded variables not fulfilling those criteria may still be important descriptors of breathlessness. To address this, independent but relevant measures could be included at the point of participant stratification or as an independent validation of group differences.

\section{Latent factors of breathlessness}

Parallels can clearly be drawn between the four factors identified in this study and our previous work despite different assessment tools being utilised and its application in a new patient group. In an investigation of breathlessness in COPD we identified the most separable factors to be what a person felt they could or could not do, how their symptoms impacted their lives and their general mood [22]. Two of these factors, corresponding to mood and perceived symptom burden, were identified in a second investigation conducted 

each disease category belonging to the higher and lower symptom burden groups shown. Each participant is represented by a single square. Colours encode disease category as follows. Red: idiopathic pulmonary fibrosis; orange: other interstitial lung disease; yellow: COPD; blue: asthma; purple: heart failure; and pink: "other" (including depression, cancer, diabetes and renal failure).
FIGURE 3 The relative proportion of

Higher symptom burden
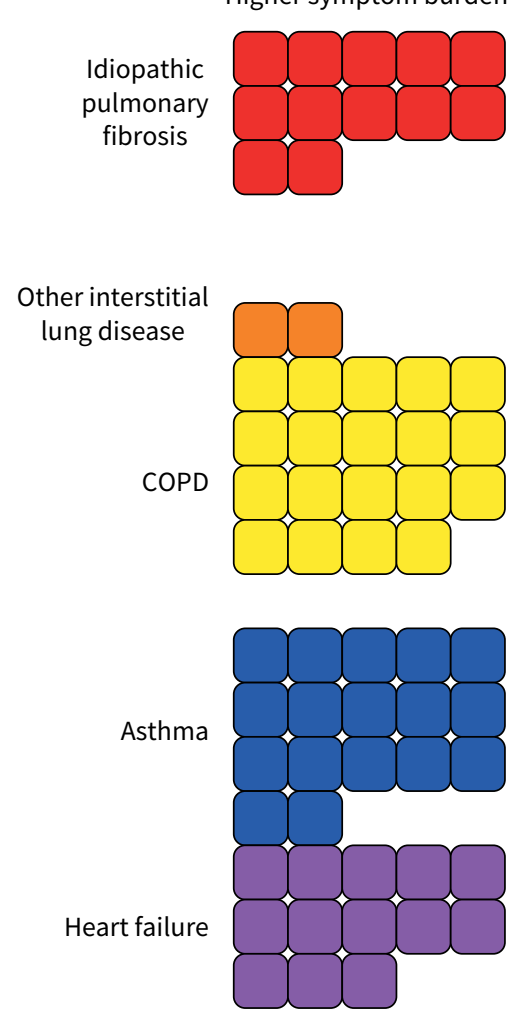

in individuals with asthma [21]. In this current work, mood/affect and symptom burden were again important factors, but here we were able to separate symptom burden into two factors; one focused on body burden (Factor 1) and a second factor relating to breathing burden (Factor 3). Interestingly, Factor 4, which contained anger/frustration measures did not collapse into Factor 2 (mood/affect), despite strong covariance. Both our previous and current work show measures contributing to factors corresponding to mood and body burden as relevant and distinct, while their strong covariance shows they are not completely independent. This illustrates the value of mechanistic research into this bi-directional relationship, which may become overlooked when investigated using other methods [8, 22, 37].

\section{Stratifying patients across diseases}

Taking scores across the four-factor model we were able to split the participant population into two groups: one corresponding to higher scores across the four factors, and one lower scoring group. Again, this is consistent to our previous work which also found a two group structure corresponding to high and low scores across four factors [21,22]. The current work extends our previous work, as group identity was found to be independent of primary disease diagnosis. This finding highlights that a common psychological reference frame for breathlessness burden could provide an opportunity to address the underlying mechanisms of breathlessness, over-come issues with comorbidities and drive treatment forwards in a more effective and personalised manner, particularly when medical therapies have been optimised. Additionally, the model could also help to contextualise other work that demonstrated a disconnect between physiological and subjective measures of breathlessness across both COPD and interstitial lung disease [10]. Stratification and cluster-based techniques have been used to good effect in other more clinically minded studies. HALDAR et al. [18] and LEUNG et al. [38] were able to identify several different asthma phenotypes using similar methods, but their works were restricted to mainly cardiorespiratory measures such as dosage of inhaled corticosteroids, neutrophil count, chemokine levels and atopy markers in a single disease. 
FIGURE 4 Factor diagram. A compact factor model in which low loading measures were sequentially removed. The retained measures are shown on the left-hand side of the diagram with the contribution of each measure to the resulting factor shown as a loading weight (rectangular boxes). Removed measures are shown in shaded boxes next to the factor to which they previously belonged. Factors are shown as ovals with descriptive labels underneath. The covariance across factors is illustrated by the curved lines.

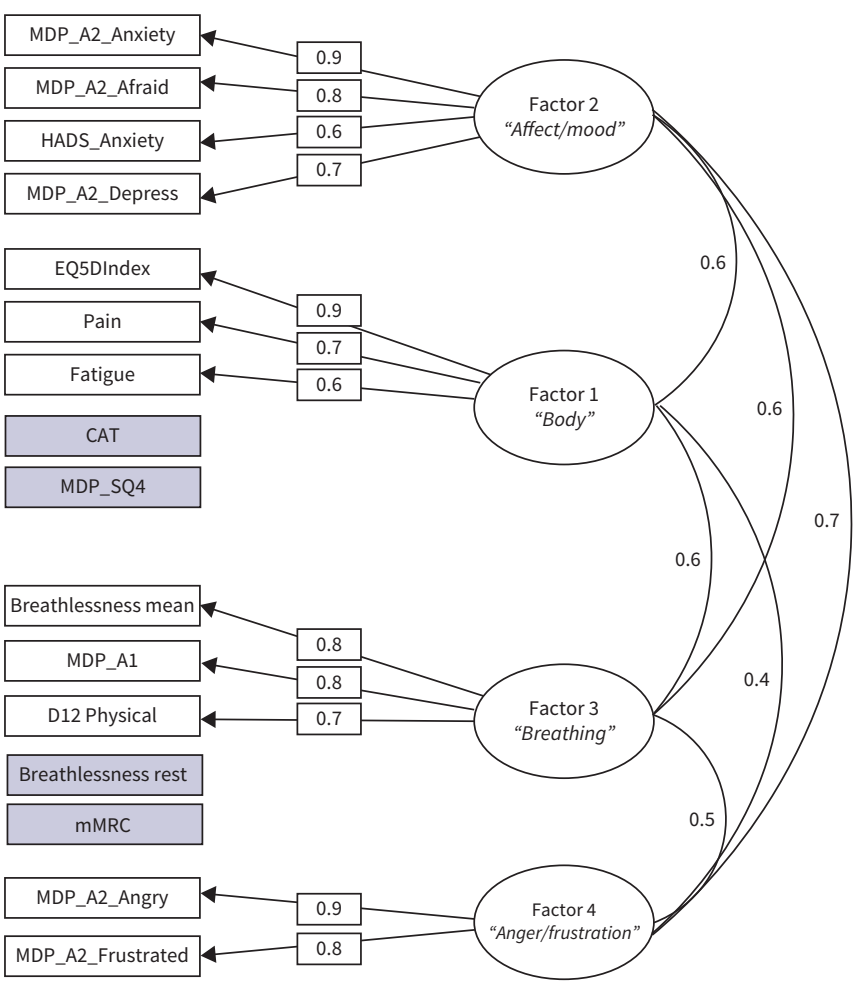

\section{A repeatable and compact measure of breathlessness}

A key requirement of any model is that it is stable across time. With this in mind we repeated our assessment of the factor structure on data acquired after six-months using confirmatory factor analysis and found that the factor structure remained stable. Having ascertained that the model was stable, we then sought to determine whether we could remove measures to create a more compact, less burdensome assessment, while maintaining a significant model fit. The iterative process of variable removal revealed only Sensory Quality 4 (SQ4 - mental breathing effort) and CAT could be removed from Factor 1 (body burden), while mMRC and breathlessness at rest could be removed from Factor 3 (breathing burden). The final compact model structure (figure 4) remained significant after testing on the six-month dataset despite the natural drop-out of participants over the 6-month period.

\section{Clinical implications}

Both the Global Initiative for Chronic Obstructive Lung Disease and American Thoracic Society statements $[3,13]$ have highlighted a clear need for a broader framework within which to describe and treat breathlessness. In introducing the rational for multidimensional models of breathlessness we highlighted the need to avoid burdening both participant and clinician with extensive questionnaires. Thus, a balance must be established, and the most relevant factors retained for clinical use. To achieve this, firstly the ability of factor scores to predict clinical outcomes should be assessed, and secondly, a randomised interventional study could target underlying factors and examine change scores across clinical outcome measures.

\section{Further considerations and limitations}

In this work, we have identified stable factors across different disease populations that we hypothesise capture important self-report aspects of the lived experience of breathlessness. However, before firm conclusions as to the utility of this model can be drawn, we must address several questions. Firstly, do different weightings across the factors link with or predict relevant outcome measures? Are there different mechanisms underlying group identity? And finally, are these groups a basis for personalised treatment pathways? To answer these questions we would need more detailed outcome measures and in depth physiological characterisation. In this work we were restricted in our ability to test for generalisability of the models. A larger sample size or second, independent dataset would have enabled us to divide the dataset into two and examine whether the models generalised to new datasets. Thus, the broad relevance of these models should be examined by future studies. Future models should also consider building in the multiple comorbidities common to patients with chronic breathlessness. In this work, we were restricted 
by sample size, and so individuals were labelled according to only their primary diagnosis, thus restricting the investigation of comorbidities influence on symptom burden. However, with a larger sample size it may be possible to examine whether particular comorbidities affect group identity or factor weightings. Additionally, physiology, duration of illness and patterns of breathlessness may be important contributors to any description of breathlessness or a relevant outcome measure, but of the measures collected, none were suitable for use across all the clinical groups. Those that were collected would have likely biased the model towards exclusively detecting disease, for example cardiac left ventricle ejection fraction would be more relevant for heart failure than asthma. Future work may consider creating disease specific longitudinal physiological burden scores, which could then be translated across different diseases. However, as demonstrated by FAISAL et al. [10], differences in physiological burden across COPD and interstitial lung disease did not explain reported breathlessness. The difficulty of incorporating physiology into such models highlights the potential for compact patient-reported tools in examining the drivers of breathlessness across disease diagnoses.

\section{Conclusions}

We have shown using machine learning techniques that a shared description of breathlessness underlies patient reports of breathlessness. These latent factors were not related to primary disease diagnosis and remained stable over time. This structure should now be investigated for clinical utility by interventional studies focused on targeted treatments for specific domains of breathlessness.

Acknowledgements: The authors extend their warm thanks to the staff conducting the study, to Hans Bornefalk and Anna Hermansson Bornefalk who made important contributions regarding the statistical aspects of the project and database management, and to all patients who participated to make this research possible.

Author contributions: S.L. Finnegan: study design for this analysis project, analysis of data, interpretation, and drafting, editing and approving manuscript. K.T.S. Pattinson: study design for this analysis project, interpretation, editing and approving manuscript, and supervision of analysis. J. Sundh, M. Sköld, C. Janson, A. Blomberg and J. Sandberg: data collection, and editing and approving manuscript. M. Ekström: original study design, data collection, interpretation, and editing and approving manuscript.

Conflict of interest: S.L. Finnegan has nothing to disclose. K.T.S. Pattinson has a UK patent application entitled "Use of cerebral nitric oxide donors in the assessment of the extent of brain dysfunction following injury" pending. J. Sundh has nothing to disclose. M. Sköld has nothing to disclose. C. Janson has nothing to disclose. A. Blomberg has nothing to disclose. J. Sandberg has nothing to disclose. M. Ekström has nothing to disclose.

\section{References}

1 Johnson MJ, Yorke J, Hansen-Flaschen J, et al. Towards an expert consensus to delineate a clinical syndrome of chronic breathlessness. Eur Respir J 2017; 49: 1602277.

2 Raman B, Cassar MP, Tunnicliffe EM, et al. Medium-term effects of SARS-CoV-2 infection on multiple vital organs, exercise capacity, cognition, quality of life and mental health, post-hospital discharge. EClinicalMedicine 2021; 31: 100683.

3 Parshall MB, Schwartzstein RM, Adams L, et al. An official American Thoracic Society statement: Update on the mechanisms, assessment, and management of dyspnea. Am J Respir Crit Care Med 2012; 185: 435-452.

4 Scano G, Gigliotti F, Stendardi L, et al. Dyspnea and emotional states in health and disease. Respir Med 2013; 107: 649-655.

5 Nishimura K, Izumi T, Tsukino M, et al. Dyspnea is a better predictor of 5-year survival than airway obstruction in patients with COPD. Chest 2002; 121: 1434-1440.

6 Ekman I, Cleland JG, Swedberg K, et al. Symptoms in patients with heart failure are prognostic predictors: insights from COMET. J Card Fail 2005; 11: 288-292.

7 Nishiyama $\mathrm{O}$, Taniguchi $\mathrm{H}$, Kondoh $\mathrm{Y}$, et al. A simple assessment of dyspnoea as a prognostic indicator in idiopathic pulmonary fibrosis. Eur Respir J 2010; 36: 1067-1072.

8 Faull OK, Hayen A, Pattinson KTS. Breathlessness and the body: Neuroimaging clues for the inferential leap. Cortex, 2017; 95: 211-221.

9 Banzett RB, O’Donnell CR, Guilfoyle TE, et al. Multidimensional dyspnea profile: an instrument for clinical and laboratory research. Eur Respir J 2015; 45: 1681-1691.

10 Faisal A, Alghamdi BJ, Ciavaglia CE, et al. Common mechanisms of dyspnea in chronic interstitial and obstructive lung disorders. Am J Respir Crit Care Med 2016; 193: 299-309.

11 O'Donnell DE, Neder JA. The enigma of dyspnoea in COPD: A physiological perspective. Respirology 2020; 25 : 134-136.

12 O’Donnell DE, Milne KM, James MD, et al. Dyspnea in COPD: New mechanistic insights and management implications. Adv Ther 2020; 37: 41-60.

13 Global Initiative for Chronic Obstructive Lung Disease, I. Global strategy for the diagnosis, management, and prevention of chronic obstructive pulmonary disease (2020 Report). 2020. https://goldcopd.org/wp-content/ uploads/2019/12/GOLD-2020-FINAL-ver1.2-03Dec19_WMV.pdf Date last updated: December 19, 2019.

14 Chekroud AM, Zotti RJ, Shehzad Z, et al. Cross-trial prediction of treatment outcome in depression: a machine learning approach. Lancet Psychiatry 2016; 3: 243-250.

$15 \mathrm{Fu} \mathrm{CH}$, Steiner H, Costafreda SG. Predictive neural biomarkers of clinical response in depression: a meta-analysis of functional and structural neuroimaging studies of pharmacological and psychological therapies. Neurobiol Dis 2013; 52: 75-83. 
Garcia-Chimeno Y, Garcia-Zapirain B, Gomez-Beldarrain M, et al. Automatic migraine classification via feature selection committee and machine learning techniques over imaging and questionnaire data. BMC Med Inform Decis Mak 2017; 17: 38.

17 Lötsch J, Ultsch A. Machine learning in pain research. Pain 2018; 159: 623-630.

18 Haldar P, Pavord ID, Shaw DE, et al. Cluster analysis and clinical asthma phenotypes. Am J Respir Crit Care Med 2008; 178: 218-224.

19 Vanfleteren LE, Spruit MA, Groenen M, et al. Clusters of comorbidities based on validated objective measurements and systemic inflammation in patients with chronic obstructive pulmonary disease. Am J Respir Crit Care Med 2013; 187: 728-735.

20 Spruit MA, Augustin IML, Vanfleteren LE, et al. Differential response to pulmonary rehabilitation in COPD: multidimensional profiling. Eur Respir J 2015; 46: 1625-1635.

21 Harrison OK, Marlow L, Finnegan S, et al. Dissociating breathlessness symptoms from mood in asthma. bioRxiv 2020; preprint [https://doi.org/10.1101/2020.07.15.204289].

22 Finnegan SL, Harrison OK, Harmer CJ, et al. Breathlessness in COPD: linking symptom clusters with brain activity. Eur Respir J 2021; in press [https://doi.org/10.1183/13993003.04099-2020].

23 Abdallah SJ, Faull OK, Wanigasekera V, et al. Opioids for breathlessness: psychological and neural factors influencing response variability. Eur Respir J 2019; 54: 1900275.

24 Ekström M, Bornefalk H, Skold M, et al. Validation of the Swedish multidimensional dyspnea profile (MDP) in outpatients with cardiorespiratory disease. BMJ Open Respir Res 2019; 6: e000381.

25 Sundh J, Bornefalk H, Skold CM, et al. Clinical validation of the Swedish version of Dyspnoea-12 instrument in outpatients with cardiorespiratory disease. BMJ Open Respir Res 2019; 6: e000418.

26 Ekström M, Bornefalk H, Skold CM, et al. Minimal clinically important differences and feasibility of Dyspnea-12 and the multidimensional dyspnea profile in cardiorespiratory disease. J Pain Symptom Manage 2020; 60: 968-975.e1.

27 Grufstedt HK, Shaker SB, Konradsen H. Validation of the COPD Assessment Test (CAT) in patients with idiopathic pulmonary fibrosis. Eur Clin Respir J 2018; 5: 1530028.

28 Yorke J, Moosavi SH, Shuldham C, et al. Quantification of dyspnoea using descriptors: development and initial testing of the Dyspnoea-12. Thorax 2010; 65: 21-26.

29 TEQ Group. EuroQol - a new facility for the measurement of health-related quality of life. Health Policy 1990; 16 199-208.

30 Baker, AM, Holbrook JT, Yohannes AM, et al. Test performance characteristics of the AIR, GAD-7 and HADS-anxiety screening questionnaires for anxiety in chronic obstructive pulmonary disease. Ann Am Thorac Soc 2018; 15: 926-934.

31 Bestall JC, Paul EA, Garrod R, et al. Usefulness of the medical research council (MRC) dyspnoea scale as a measure of disability in patients with chronic obstructive pulmonary disease. Thorax 1999; 54: 581-586.

32 Costello $\mathrm{AB}$, Osborne J. Best practices in exploratory factor analysis: Four recommendations for getting the most from your analysis. Pract Assess, Res, Eval 2005; 10: 7.

33 Schreiber JB, Nora A, Stage EK, et al. Reporting structural equation modeling and confirmatory factor analysis results: a review. J Educ Res 2006; 99: 323-338.

34 Zygmont C, Smith M. Robust factor analysis in the presence of normality violations, missing data, and outliers: Empirical questions and possible solutions. Quant Methods Psychol 2014; 10: 40-55.

35 Saraçli S, Doğan N, Doğan İ. Comparison of hierarchical cluster analysis methods by cophenetic correlation. Inequ Appl 2013; 2013: 203.

36 Fraley C, Raftery AE. Model-based clustering, discriminant analysis, and density estimation. J Am Stat Assoc 2002; 97: 611-631.

37 Van den Bergh O, Witthöft M, Petersen S, et al. Symptoms and the body: Taking the inferential leap. Neurosci Biobehav Rev 2017; 74: 185-203.

38 Leung TF, Wong GW, Ko FW, et al. Clinical and atopic parameters and airway inflammatory markers in childhood asthma: a factor analysis. Thorax 2005; 60: 822-826. 